\title{
Philosophiques
}

\section{Le spectre épistocratique}

\section{Jessy Giroux}

Volume 40, numéro 2, automne 2013

\section{Autorité démocratique et contestation}

URI : https://id.erudit.org/iderudit/1023699ar

DOI : https://doi.org/10.7202/1023699ar

Aller au sommaire du numéro

\section{Éditeur(s)}

Société de philosophie du Québec

ISSN

0316-2923 (imprimé)

1492-1391 (numérique)

Découvrir la revue

\section{Citer cet article}

Giroux, J. (2013). Le spectre épistocratique. Philosophiques, 40(2), 301-319. https://doi.org/10.7202/1023699ar

\section{Résumé de l'article}

J'aborde dans cet article un problème que je nomme le "spectre épistocratique ». Le problème se présente ainsi : s'il existe des vérités politiques, c'est-à-dire des positions politiques qui soient véritablement bonnes, ne devrait-on pas faire de l'atteinte de ces vérités politiques l'objectif central de notre système politique, ce qui pourrait nous conduire à limiter le pouvoir populaire afin de laisser les individus « éclairés " prendre toutes les décisions politiques ? J'explore différentes stratégies possibles afin de défendre la démocratie contre l'attrait d'une telle épistocratie, et je conclus que la meilleure stratégie consiste à souligner qu'une démocratie délibérative bien organisée démontre un potentiel épistémique supérieur à tout autre système politique. Je termine en montrant comment une telle conclusion parvient à nous informer sur le phénomène de la contestation politique.
Ce document est protégé par la loi sur le droit d'auteur. L'utilisation des services d'Érudit (y compris la reproduction) est assujettie à sa politique d'utilisation que vous pouvez consulter en ligne.

https://apropos.erudit.org/fr/usagers/politique-dutilisation/ 


\title{
Le spectre épistocratique
}

\author{
JESSY GIROUX \\ University of Toronto \\ jessy.giroux@mail.utoronto.ca
}

\begin{abstract}
RÉSUMÉ. - J'aborde dans cet article un problème que je nomme le «spectre épistocratique». Le problème se présente ainsi: s'il existe des vérités politiques, c'est-à-dire des positions politiques qui soient véritablement bonnes, ne devrait-on pas faire de l'atteinte de ces vérités politiques l'objectif central de notre système politique, ce qui pourrait nous conduire à limiter le pouvoir populaire afin de laisser les individus «éclairés» prendre toutes les décisions politiques? J'explore différentes stratégies possibles afin de défendre la démocratie contre l'attrait d'une telle épistocratie, et je conclus que la meilleure stratégie consiste à souligner qu'une démocratie délibérative bien organisée démontre un potentiel épistémique supérieur à tout autre système politique. Je termine en montrant comment une telle conclusion parvient à nous informer sur le phénomène de la contestation politique.
\end{abstract}

\begin{abstract}
In this paper, I consider a problem that I call the "epistocratic specter". The problem goes as follows: if there are political truths, by which I mean political positions that are truly good, shouldn't we make the attainment of such truths the central goal of our political system, which could lead us to restrict popular power and let only truly "enlightened" individuals make every political decision? I explore various possible strategies in order to defend democracy against the appeal of such an epistrocratic system, and I conclude that the best available strategy is one that emphasizes that a well-organized deliberative democracy possesses an epistemic potential that is superior to that of any other political system. I conclude by showing how this fact can inform our understanding of the phenomenon of political dissent.
\end{abstract}

\section{Contestation, démocratie et vérité politique}

En philosophie, on s'intéresse généralement au phénomène de la contestation politique dans la mesure où celui-ci soulève, et surtout incarne, le problème fondamental de la légitimité politiq des limites de l'autorité étatique. En effet, indépendamment de la nature spécifique et de l'étendue de ses revendications, le contestataire ${ }^{1}$ interpelle toujours implicitement le gouvernant sur ce lien étroit qui unit volonté populaire et gouverne légitime. Qu'il en appelle au renversement complet d'un régime politique, comme avec les révoltes du Printemps arabe; qu'il en appelle à un virage idéologique profond des gouvernements, comme avec le mouvement d'Occupy Wall Street; ou qu'il interpelle le parti au pouvoir sur un enjeu beaucoup plus circonscrit, comme le firent les associations étudiantes québécoises lors du Printemps Érable, le contestataire vient immanquablement rappeler au gouvernant que le peuple

1. L'usage du masculin vise uniquement à alléger le texte.

PHILOSOPHIQUES 40/2 - Automne 2013, p. 301-319 
est sa source et sa raison d'être, le début et la fin de son existence. Lorsqu'on s'attarde au contexte des démocraties représentatives, le phénomène de la contestation soulève des questionnements philosophiques plus spécifiques. On se demandera par exemple dans quelle mesure la contestation perd de sa légitimité lorsqu'un gouvernement respecte le mandat pour lequel il a été élu, ou inversement, dans quelle mesure la contestation devient un devoir citoyen lorsqu'un gouvernement s'écarte de son mandat, et à quel moment cet écart justifie un renversement du pouvoir en place.

Dans le cadre du présent article, je souhaite aborder un problème philosophique qui s'éloigne quelque peu de ces enjeux traditionnellement associés au phénomène de la contestation politique. La nature inusitée de mon enquête s'explique surtout par le fait qu'elle soit largement motivée par des enjeux propres à la méta-éthique, à savoir le problème du relativisme moral et la possibilité de vérités objectives dans le domaine du normatif. Dans cette optique, mon intérêt pour le phénomène de la contestation politique tient principalement à ce que l'action du contestataire présuppose chez celui-ci l'acceptation de ce que j'appellerai une "vérité politique». Dans «vérité politique», j'entends par politique la sous-catégorie du normatif qui concerne les décisions collectives, et j'entends par vérité l'idée qu'une position $^{2}$ politique X puisse être considérée comme étant «la bonne » position politique à adopter. Le concept se veut analogue au concept de vérité morale; une proposition politico-économique telle que " on doit répartir la richesse » a une prétention à la vérité analogue à la proposition morale «on doit tenir ses promesses ».

Je soutiens qu'on ne peut, de manière cohérente, être contestataire sans reconnaître par le fait même une forme de vérité politique - à savoir la vérité de ce pourquoi l'on conteste. On pourrait objecter qu'il est possible de contester une position politique $\mathrm{X}$ tout en ne défendant pas d'autre option claire. Il est possible, par exemple, de protester contre un mode de scrutin uninominal à un tour, ou contre un impôt à taux unique sans pour autant défendre ou même connaître une solution préférable. Cependant, même dans de tels cas, le contestataire reconnaît l'existence de vérités politiques de manière négative, c'est-à-dire qu'en s'opposant à une position politique $\mathrm{X}$, le contestataire soutient que X n'est pas la bonne position politique. Or soutenir que $\mathrm{X}$ n'est pas la bonne position politique revient à admettre une vérité politique, à savoir la vérité de non-X. Le fait que le contestataire ne sache pas quelle version de non-X est préférable n'enlève rien au fait qu'il reconnaisse que $\mathrm{X}$ n'est pas la bonne position politique. Cette forme de

2. J'utiliserai principalement le concept de «position politique» dans cet article. Ce concept englobe aussi bien les politiques (policies) adoptées par les gouvernements que les principes ou théories politiques défendus par les différents acteurs politiques. J'emploierai également le concept de «décision politique » pour référer aux positions politiques qui sont adoptées par les gouvernants et les assemblées. 
reconnaissance négative est donc la manière minimale par laquelle toute contestation politique présuppose l'acceptation d'une vérité politique.

Or, dès lors que l'on reconnaît l'existence de vérités politiques, on ouvre la boîte de Pandore et l'on se trouve confronté à ce que j’appelle le "spectre épistocratique ». Le spectre épistocratique se présente de la manière suivante: s'il existe effectivement des vérités politiques, il semble naturel de croire que celles-ci constituent un des objectifs principaux, sinon l'objectif principal des systèmes politiques. C'est presque une vérité de La Palice que de soutenir qu'on attend d'un gouvernement qu'il prenne de bonnes décisions c'est-à-dire, pour reprendre notre terminologie, des décisions conformes aux vérités politiques. Or la démocratie paraît très souvent constituer un obstacle à l'atteinte de ces vérités politiques; nul besoin d'être un cynique ou un élitiste platonicien pour reconnaître que les opinions populaires tantôt extrémistes, tantôt incohérentes que révèlent certaines enquêtes sont autant d'indices soulignant le risque épistémique associé à un système démocratique. Or, si l'atteinte de vérités politiques constitue réellement l'objectif central de tout système politique, ne devrait-on pas préférer un système épistocratique, c'est-à-dire un système qui restreint le pouvoir populaire dans le but de favoriser l'atteinte de vérités politiques ? ${ }^{3}$ En quoi la démocratie, marquée par son déficit épistémique, est-elle préférable à une épistocratie? Tel est le spectre épistocratique qui apparaît dès lors que l'on admet l'existence de vérités politiques.

Mon but ici est de proposer une défense de la démocratie. Pour ce faire, j'évaluerai dans les sections suivantes différentes stratégies argumentatives qui peuvent être utilisées afin de fonder la préférabilité de la démocratie sur l'épistocratie. Je distinguerai essentiellement trois grandes stratégies: la première consiste à rejeter la pertinence même du problème en niant (2) qu'il existe de quelconques vérités politiques, une stratégie que j’appelle nihilisme. La seconde consiste à démontrer (3) que la valeur centrale de la démocratie, à savoir l'égalité, est supérieure à la valeur centrale de l'épistocratie, à savoir la vérité, ce qui justifie un trade-off entre égalité et vérité. La troisième consiste à nier une autre prémisse du problème en soutenant (4) que la démocratie, en dépit de certaines apparences, a en réalité une valeur épistémique supérieure à tout autre système politique. Je conclurai $(5)$ que la troisième stratégie est la plus prometteuse si l'on cherche à fonder la supériorité du système démocratique, et je discuterai rapidement de ce que cela peut nous enseigner sur le phénomène de la contestation.

3. Un système épistocratique peut prendre différentes formes, mais se présente généralement comme une "gouverne des sages", ce qui peut consister en une restriction du droit de vote aux seuls citoyens suffisamment éduqués ou à tout autre procédure garantissant que seule l'élite intellectuelle ait un pouvoir décisionnel en matière politique. Voir Estlund 2008, chapitre XI: «Why Not an Epistocracy of the Educated?".

4. Faute d'un équivalent réellement satisfaisant en français, j'emploierai dans cet article le terme anglais «trade-off». 


\section{Le nihilisme ou quasi-nihilisme}

La première stratégie envisageable afin de fonder la préférabilité de la démocratie par rapport à l'épistocratie consiste tout simplement à retirer à cette dernière ce qui justifie son attrait, à savoir sa valeur épistémique ou sa prétendue capacité à mener aux bonnes décisions politiques. On peut envisager différentes formes plus ou moins radicales de cette stratégie. Je considérerai ici deux versions: la première consiste en un discrédit total de la notion de vérité politique (2.I) et la seconde consiste en une réinterprétation de cette notion (2.2). Dans le premier cas, je parlerai de nihilisme pur et dur, et, dans le second, je parlerai plutôt de quasi-nihilisme.

\subsection{Le nihilisme}

J'ai souligné à la section I que l'acte de contestation présupposait la croyance en des vérités politiques, pour la simple raison que le contestataire reconnaît à tout le moins que la position politique qu'il conteste n'est pas la bonne position politique. Or il est possible que cette croyance soit en réalité une simple erreur, ou une illusion. Bien que toutes nos actions politiques reposent sur cette idée que certaines positions politiques sont bonnes et d'autres mauvaises, une telle idée est en réalité fautive, car il n'existe ultimement aucune position politique qui puisse réellement — c'est à dire objectivement — être dite supérieure à une autre.

Une telle thèse peut être défendue de différentes façons. On pourrait par exemple soutenir, à la manière des non-cognitivistes en méta-éthique, que les énoncés politiques, compris comme sous-catégorie du normatif, n'ont tout simplement aucune valeur de vérité puisqu'ils n'ont pas pour fonction d'exprimer une quelconque croyance concernant un état de choses. Dans sa forme la plus simple et traditionnelle, cette théorie soutient que, lorsqu'un individu défend une position politique, ce qu'il fait réellement revient à exprimer une émotion, ou un sentiment d'approbation ou de désapprobation face à un enjeu ${ }^{5}$. Ainsi, un contestataire qui soutiendrait qu'il ne faut pas réduire le financement des programmes sociaux afin de ne pas nuire aux plus démunis devrait tout simplement être compris comme exprimant une émotion négative concernant le sort des plus démunis de la société. S'inspirant d'une telle théorie, on pourrait tenter de couper l'herbe sous le pied aux épistocrates en discréditant l'idée même qui leur accorde une certaine crédibilité, à savoir l'idée de vérité politique, en soutenant que les énoncés politiques n’ont tout simplement pas pour fonction de représenter des états de choses.

Le problème avec ce type de stratégie - un problème que partagent toutes les formes de non-cognitivisme, tant l'émotivisme traditionnel que 
des versions plus complexes ${ }^{6}$ - est que l'expérience courante tend à contredire l'idée que les énoncés politiques n'exprimeraient aucune croyance. Le contestataire qui s'oppose aux compressions budgétaires dans les programmes sociaux le fait normalement sur la base d'une croyance concernant le caractère injuste d'une telle politique. L'individu qui s'engage politiquement a généralement une vision du type de société qui lui paraît être juste, approprié, voir idéal. Il ne fait pas qu'exprimer son approbation ou désapprobation: il décrit des états de choses comme possédant ou non certaines propriétés (juste, bien, nécessaire, etc.), et détermine la "bonne position» comme étant celle qui correspond à cet état de choses. Bref, en s'opposant à ce qu'on pourrait appeler la phénoménologie politique, le non-cognitiviste se trouve confronté à un fardeau on ne peut plus lourd.

Afin d'éviter d'avoir à remettre en cause l'expérience courante, on pourrait préférer se tourner vers une autre stratégie nihiliste ${ }^{7}$ qui consiste à reconnaître que les énoncés politiques expriment des croyances, mais à soutenir que ces croyances sont systématiquement fausses, pour la simple et bonne raison qu'il n'existe aucune propriété correspondant à ces croyances. Ainsi, lorsque notre contestataire affirme qu'il est mal (ou injuste, ou cruel, etc.) de pénaliser les plus démunis en réduisant les budgets des programmes sociaux, il exprime une croyance qui est nécessairement fausse, puisqu'il n'existe aucune propriété dans le monde qui corresponde au terme «mal» (ou injuste ou cruel). Une telle "théorie de l'erreur ${ }^{8}$ » laisse ainsi la phénoménologie politique intacte et s'attaque plutôt à la métaphysique qui soustend cette phénoménologie, métaphysique jugée suspecte. Puisqu'elle ne s'oppose pas à l'expérience courante et qu'elle affirme se ranger dans le camp de la parcimonie, cette forme de nihilisme semble offrir la meilleure façon de s'opposer à l'épistocratie en discréditant la notion de vérité politique.

L'acceptation d'un nihilisme aussi radical que celui considéré ici comporte cependant un coût énorme, que peu accepteront d'assumer: en rejetant l'idée même de vérité politique, on perd de ce fait le fondement rationnel nous permettant de dénoncer non-arbitrairement diverses atrocités. Si nos concepts normatifs sont dépourvus de tous référents pertinents, et si toute prétention à la validité normative repose ultimement sur une erreur, on ne possède alors aucun outil pour démontrer le caractère inacceptable d'un génocide, d'un système esclavagiste, etc. On pourra bien sûr continuer à combattre de telles atrocités, mais on ne pourra guère, de manière cohérente,

6. Pour une comparaison des différentes formes de non-cognitivisme, voir Darwall, Gibbard et Railton (I992), p. I44-I 52.

7. Ou «anti-réaliste» pour employer un terme plus courant en philosophie morale contemporaine.

8. Voir notamment Mackie (1977). 
prétendre que notre cause est plus noble ou plus juste que celle du camp adverse.

Même en supposant qu'un philosophe soit prêt à assumer les conséquences d'une telle position, on constate rapidement qu'un nihilisme aussi radical ne parvient tout simplement pas à remplir le mandat qui lui est confié, pour la simple raison qu'en rejetant les fondements mêmes de la validité normative une telle théorie, bien que révoquant la justification d'un système épistocratique, rend tout aussi impossible la justification d'un système démocratique. En effet, l'impotence justificatrice à laquelle conduit le nihilisme ne se limite pas aux positions politiques à proprement parler, mais s'étend également aux systèmes politiques eux-mêmes; s'il est impossible d'affirmer sans erreur que la défense des plus démunis de la société est une cause bonne (ou juste, ou noble, etc.), il est tout aussi impossible d'affirmer sans erreur que la démocratie est un système bon (ou juste, ou supérieur, etc.).

On pourrait tenter de contourner ce problème en faisant reposer la supériorité de la démocratie sur des énoncés entièrement descriptifs capables de justification, contrairement aux énoncés évaluatifs. On pourrait par exemple dire que la supériorité de la démocratie tient au fait qu'elle permet, plus que tout autre système, d'accomplir une égalité entre les citoyens, ce qui constitue un énoncé descriptif évitant le piège propre aux énoncés évaluatifs. Une telle stratégie est cependant vouée à l'échec, puisqu'on ne pourra éviter d'en arriver tôt ou tard à faire reposer ces descriptions sur des évaluations, et on retombera alors dans le piège décrit précédemment. Par exemple, après avoir tenté de fonder la valeur de la démocratie sur sa capacité à accomplir une égalité entre les citoyens, il nous faudra encore considérer la prémisse évaluative selon laquelle l'égalité est bien (ou juste, ou désirable), et il nous faudra également démontrer que l'atteinte de l'égalité est une fin supérieure à d'autres fins susceptibles d'être visées par un système politique, ce qui requerrait également l'usage d'énoncés évaluatifs.

Bref, la stratégie nihiliste ne parvient tout simplement pas à remplir adéquatement sa mission. En discréditant complètement la notion de vérité politique, elle supprime par le fait même toute possibilité de justifier la supériorité de la démocratie. Faire appel au nihilisme pour critiquer l'épistocratie revient donc à jeter le bébé, et toute la pouponnière, avec l'eau du bain.

\subsection{Le quasi-nihilisme, ou la vérité procédurale}

On peut cependant concevoir une version plus modérée de l'argument nihiliste, que l'on pourrait qualifier de quasi-nihilisme, lequel permet de discréditer l'épistocratie tout en préservant une forme de justification normative. La stratégie consiste ici non pas à rejeter entièrement la notion de vérité politique, mais plutôt à rejeter l'idée que ces vérités seraient indépendantes des individus. Les vérités politiques seraient en réalité constituées, créées par les acteurs politiques. 
Une telle stratégie amène généralement à interpréter la notion de vérité politique en termes procéduraux: une décision politique peut être dite bonne si et seulement si elle est le résultat d'une procédure acceptable. Cet argument ne doit pas être confondu avec la thèse du procéduralisme épistémique, selon laquelle la délibération démocratique est le meilleur moyen de tendre vers la vérité, conçue comme indépendante de la procédure9 ${ }^{9}$. La thèse considérée ici est beaucoup plus forte et consiste, non pas à dire que l'atteinte de la vérité devient "plus probable » grâce à la délibération, mais plutôt que la vérité est carrément constituée par la délibération. Ce qui est politiquement vrai est ce qui est accepté dans le cadre d'une (bonne) procédure délibérative. On cherche donc ici à faire abstraction de toute valeur extérieure à la délibération afin de justifier les décisions politiques. Une telle approche correspond à la définition que Brian Barry donne du constructivisme: "[Constructivism is] the doctrine that what would be agreed on in some specified kind of situation constitutes justice» (Barry, I989, 268).

Cette stratégie quasi-nihiliste ou constructiviste paraît efficace pour retirer tout attrait à l'épistocratie, car si la vérité politique est effectivement un produit procédural, on n'a alors plus aucune raison de restreindre le pouvoir populaire et de confier ce pouvoir aux mains de prétendus experts (ou sages) capables de guider la société vers les vérités politiques. Nul ne peut prétendre être un expert si les vérités politiques sont constituées par les procédures. L'épistocratie deviendrait ainsi un système absurde, fondé sur une incompréhension de la nature des vérités politiques.

Bien qu'elle parvienne efficacement à discréditer l'épistocratie, une telle conception de la vérité politique pose cependant plusieurs problèmes, à commencer par l'arrivée d'une forme de relativisme: les décisions radicalement opposées de deux groupes délibératifs devront toutes deux être jugées bonnes si ces délibérations sont effectuées selon les règles de l'art. On ouvre ainsi la porte à la validation de positions extrêmes, dans la mesure où une assemblée constituée de personnes partageant les mêmes positions racistes (ou sexistes, etc.) en arriveraient à un consensus sur de telles positions - qui devraient alors être considérées comme bonnes. Il est cependant possible de résoudre un tel problème en spécifiant les termes de la délibération afin d'inclure les points de vue de toute personne potentiellement affectée par la déci$\operatorname{sion}^{10}$. On devrait ainsi inclure le point de vue des minorités ethniques ou sexuelles si l'on souhaitait délibérer sur des enjeux qui les concernent.

Un problème plus sérieux demeure cependant quant à la tentative de faire complètement fi de toute vérité politique qui n'est pas un produit procédural, ce que David Estlund nomme "the flight from substance» (Estlund, 2008, 65). C'est là une tentative qui semble vouée à l'échec. En effet, peu importe la façon dont on interprétera la valeur justificatrice inhérente à la

9. Voir section 4.

10. Voir par exemple la procédure décrite par Thomas Scanlon (I998). 
procédure - qu'il s'agisse d'agrégation des préférences, de prise en considération des points de vue de toutes les personnes concernées, ou de l'abstraction des intérêts particuliers comme dans la procédure rawlsienne (Rawls, I985) -, cette valeur procédurale sera elle-même en attente de justification:

No adequate distinction between purely procedural values, on the one hand, and substantive values, on the other, can explain on a purely procedural basis why political outcomes ought to answer to citizens' interests or values or choices at all (Estlund, 2008, 28).

La procédure doit pouvoir être justifiée sur la base de valeurs non procédurales, c'est-à-dire de vérités politiques indépendantes de toute procédure $^{11}$. En l'absence de telles vérités non procédurales, on ne sait tout simplement pas pourquoi l'on devrait avoir recours à la procédure, et on est également incapable de discréditer certaines règles procédurales qui paraissent farfelues ou injustes. Or, s'il est impossible d'éliminer toute référence à des vérités non procédurales, c'est la stratégie quasi-nihiliste qui s'écroule par le fait même. En effet, s'il existe des vérités politiques indépendantes de la procédure, on est alors incapable de faire disparaître le spectre épistocratique, dans la mesure où l'existence de telles vérités ouvre la porte à la nécessité de points de vue d'experts.

Peut-être est-il possible pour un quasi-nihiliste d'admettre l'existence de vérités non procédurales sans pour autant être à la merci du spectre épistocratique. Par exemple, on pourrait soutenir que le rôle des experts est extrêmement circonscrit, se limitant à la détermination de règles procédurales à partir d'un nombre restreint de vérités politiques non procédurales. En soulignant ainsi la portée limitée des vérités non procédurales, on pourrait tenter de retirer son attrait à l'épistocratie ${ }^{12}$. Une telle stratégie me semble cependant peu prometteuse, pour la raison suivante: si l'on souhaite reconnaître l'existence de vérités politiques non procédurales tout en limitant leur portée, il nous faut expliquer pourquoi ces vérités ont une portée

11. Pour la suite du texte, j'emploierai l'expression "vérité procédurale » pour parler de toute position politique dont la vérité provient de l'acceptation dans le cadre d'une bonne procédure. La vérité procédurale s'oppose ainsi à la vérité non procédurale (ou vérité politique non procédurale), cette dernière correspondant à une position politique dont la vérité est indépendante de toute procédure. Enfin, une vérité procédurale ne doit pas être confondue avec une vérité portant sur la procédure: un énoncé du type "il faut prendre en considération le point de vue de toutes les personnes concernées" est une vérité portant sur la procédure — dans la mesure où elle permet de déterminer ce qui constitue ou non une bonne procédure - mais elle n'est pas (nécessairement) une vérité procédurale, puisque sa vérité ne découle pas (nécessairement) de son acceptation dans le cadre d'une bonne procédure. Cette dernière distinction deviendra vite importante dans la discussion qui suit.

12. Il ne s'agirait cependant plus à proprement parler d'une position quasi-nihiliste, puisqu'on reconnaitrait l'existence de vérités politiques qui ne sont pas de simples produits procéduraux. On pourrait sans doute parler de quasi-quasi-nihilisme. 
limitée. On peut alors concevoir deux stratégies différentes afin d'expliquer cette portée limitée, et toutes deux semblent vouées à l'échec.

La première stratégie consiste à soutenir que les seules vérités politiques non procédurales qui existent sont des vérités permettant de déterminer la bonne procédure. Ainsi, seuls les énoncés du type «il faut prendre en considération le point de vue de toutes les personnes concernées» seraient admis au rang de vérités politiques non procédurales. Tout type d'énoncé normatif qui ne porte pas sur la procédure elle-même, par exemple «il faut aider les plus démunis de la société », ne pourrait être dit vrai que dans la mesure où il serait admis dans le cadre d'une bonne procédure. Pour résumer en une formulation on ne peut plus chargée, cette stratégie consiste à nier l'existence de vérités non procédurales qui ne portent pas sur la procédure.

Le problème de cette première stratégie est qu'il paraît tout simplement invraisemblable que les seules vérités politiques non procédurales existantes soient des énoncés portant sur la bonne procédure. Cela est invraisemblable si l'on considère l'étroite similarité entre ce type d'énoncés et les énoncés politiques qui ne portent pas sur la procédure. Si l'on compare par exemple les deux types d'énoncés considérés ci-dessus - «il faut prendre en considération le point de vue de toutes les personnes concernées» et «il faut aider les plus démunis de la société » - on constate que tous deux semblent tirer leur validité de leur nature intuitive, c'est-à-dire de leur caractère évident en soi $^{13}$. Ils soulèvent également le même type de problèmes épistémologique et métaphysique que ceux associés aux énoncés normatifs ou prescriptifs. Bref, la similarité entre ces types d'énoncés est telle qu'il nous faudrait apparemment de bonnes raisons pour admettre que seul l'un d'eux puisse aspirer à la validité normative. Or le fait que seul l'un des énoncés (le premier) puisse permettre de déterminer ce qui constitue une bonne procédure est un type de considération qui ne semble tout simplement pas pertinent; ce n'est pas là le type de raison qui paraît justifier l'idée que seul un des types d'énoncés puisse prétendre être vrai. Pour cette raison, il paraît invraisemblable que les seules vérités politiques non procédurales qui existent soient des vérités portant sur la procédure. Dès lors que l'on ouvre la porte aux vérités non procédurales, on semble condamné à admettre que ces vérités puissent porter sur un large éventail d'objets.

La deuxième stratégie consiste à reconnaître l'existence de vérités non procédurales qui ne portent pas sur la procédure, en ajoutant cependant que, pour une raison ou une autre, ces vérités n'ont pas à être prises en considération. Ainsi, un énoncé du type «il faut aider les plus démunis de la société » pourrait être considéré comme non procéduralement vrai ${ }^{14}$ sans pour autant être jugé important ou pertinent. L'argument consiste donc ici à

13. Voir Audi, 2008, pour une description de la justification intuitive d'énoncés moraux. 14. C'est-à-dire comme étant une vérité non procédurale. 
soutenir que les seules vérités politiques qui importent sont les vérités procédurales. On pourrait par exemple affirmer que seules les vérités procédurales sont légitimes puisqu'elles sont les seules choisies par les agents concernés. Les vérités non procédurales seraient de moindre importance précisément en raison de leur caractère externe par rapport aux agents concernés. Une telle stratégie parviendrait ainsi à retirer son attrait au système épistocratique, dans la mesure où l'importance accordée à la position des experts ou des sages reflète l'importance accordée aux vérités non procédurales.

Le problème de cette deuxième stratégie est similaire au problème soulevé dans le cas de la première stratégie: il paraît tout simplement invraisemblable que les vérités non procédurales qui ne portent pas sur la procédure puissent être jugées non pertinentes ou sans importance. Dans bien des cas, la prise en considération de ces vérités est tout simplement incontournable. Par exemple, certaines positions tantôt extrêmes (par ex. autoriser le meurtre récréatif), tantôt collectivement auto-destructrices (par ex. autoriser l'annulation de contrats sans raison valable) sont telles qu'on ne peut faire autrement que de leur accorder une certaine importance ou pertinence - et on ne saurait tenir pour bonne la décision d'une assemblée qui incorporerait de telles positions, même si la procédure s'effectue dans les règles de l'art. Les vérités non procédurales de ce type ont une force contraignante inévitable sur ce qui peut ou non être considéré comme une bonne décision politique. Au-delà des exemples de positions extrêmes ou auto-destructrices, certaines politiques peuvent tout simplement être moins efficaces que d'autres dans l'atteinte d'objectifs communs, et l'adoption par une assemblée de telles politiques sous-optimales pourrait difficilement être qualifiée de bonne. Ce sont là des exemples parmi d'autres illustrant la difficulté, voire l'impossibilité de n'accorder d'importance qu'aux vérités procédurales ${ }^{15}$.

Les deux stratégies considérées afin de préserver l'argument quasinihiliste conduisent donc toutes deux à des positions invraisemblables, et, tout comme le nihilisme pur et dur, le quasi-nihilisme paraît tout simplement incapable d'éviter le spectre épistocratique. On se tournera donc vers une autre stratégie, qui consiste non pas à discréditer ou modifier la notion de vérité politique, mais plutôt à présenter celle-ci comme étant de second ordre par rapport à la valeur qu'incarne la démocratie, à savoir la valeur d'égalité.

\section{La primauté de l'égalité}

Il a été dit en introduction que l'on pouvait concevoir le choix de la démocratie comme étant l'acceptation d'un trade-off entre égalité et vérité: préférer la

15. On pourrait répondre ici qu'une procédure véritablement bonne permettrait d'éviter ce type de mauvaises décisions politiques. Or c'est là un type d'argument d'une autre nature que je considère - et appuie — à la section 5 . 
démocratie à l'épistocratie, c'est sacrifier un peu de bonnes décisions politiques - c'est-à-dire des décisions conformes aux vérités politiques — afin d'obtenir un peu plus d'égalité entre citoyens. Les stratégies nihiliste et quasi-nihiliste considérées à la section précédente cherchent essentiellement à rejeter cette idée de trade-off, et ce, en discréditant ou en modifiant la notion de vérité politique; puisque la vérité politique est illusoire (nihilisme) ou créée par la procédure (quasi-nihilisme), il n'y a pas de véritable sacrifice de la vérité politique qui découle du choix de la démocratie.

La stratégie que je souhaite considérer dans cette section est différente de la stratégie nihiliste en ce qu'elle reconnaît l'existence de vérités politiques et reconnaît également l'existence d'un trade-off entre égalité et vérité résultant du choix de la démocratie. Plutôt que de nier l'existence d'un trade-off, on cherchera plutôt à justifier celui-ci en soulignant la valeur supérieure de l'égalité qui est réalisée par la démocratie. L'argument se présente de la manière suivante: s'il est vrai qu'octroyer le vote à tous les citoyens, indépendamment de leur niveau d'éducation ou de leurs connaissances politiques, augmente le risque de mauvaises décisions politiques - que ce soit directement, par la voie de référendums, ou indirectement, par l'élection de gouvernements incompétents, opportunistes ou extrémistes -, il s'agit là d'un moindre mal par rapport à un système qui briserait l'égalité citoyenne en n'accordant le droit de vote qu'à une minorité éduquée. Plus abstraitement, cela revient à affirmer la supériorité ou la primauté axiologique de la valeur d'égalité politique, par rapport à la valeur de vérité politique.

Le défi qui attend une telle stratégie est bien sûr de parvenir à une démonstration acceptable de la supériorité de l'égalité politique par rapport à la vérité politique. Or c'est là un défi qui est beaucoup moins évident qu'il ne le paraît à première vue. En effet, comment peut-on comparer deux entités aussi abstraites et déterminer que l'une d'elles est supérieure à l'autre? Il va sans dire que nous ne possédons aucune forme d'unité de mesure nous permettant de comparer des entités abstraites de ce type. Or, pour parvenir à fonder in abstracto la supériorité d'une valeur sur une autre, il nous faut sinon une unité de mesure, du moins une explication plausible de cette supériorité. Malheureusement, aucune explication concevable ne paraît véritablement efficace. Pour illustrer le principal problème qui guette ce type de démonstrations in abstracto, je considère ici rapidement un exemple que l'on retrouve dans The Constitution of Equality: Democratic Authority and its Limits de Thomas Christiano (Christiano, 2008).

Dans le premier chapitre de cet ouvrage, Christiano se livre à une enquête des sources de l'autorité démocratique et propose un modèle résolument fondationnaliste de la démocratie qui peut être interprété comme une défense de la primauté axiologique de la valeur d'égalité. Selon Christiano, l'autorité de la démocratie doit être comprise comme reposant sur sa réalisation d'un principe d' "égalité publique " de tous les citoyens, principe qui repose à son tour sur un principe plus abstrait d' "égalité ", laquelle est 
pour sa part interprétée dans le sens de "dignité humaine », et enfin doit être comprise dans le sens de "valeur intrinsèque ». Dire des hommes qu'ils sont égaux revient, selon Christiano, à dire qu'ils ont une égale dignité, qui revient à son tour à dire qu'ils sont les seuls êtres capables de percevoir et de créer des valeurs intrinsèques, ce fait leur accordant du même coup une valeur intrinsèque: "Equal status is based on the fact that human beings all have essentially the same basic capacities to be authorities in the realm of value (Christiano, 2008, I7).

Selon Christiano, donc, l'égalité émane en quelque sorte de l'humanité au niveau le plus primaire, élémentaire, que l'on puisse concevoir. Aucune autre valeur ne peut prétendre jouir d'un statut comparable. C'est pour cette raison que la réalisation de l'égalité doit être l'objectif premier de tout système politique, et pour cette raison que la démocratie, qui est fondée sur le principe d'égalité, est préférable à tout autre système politique, y compris l'épistocratie. Il y a donc ici une véritable tentative de fonder in abstracto la supériorité de la valeur d'égalité.

Le problème de cette démonstration de Christiano, un problème qui guette toute tentative de ce type, est que ce qu'on appelle une démonstration n'est en réalité qu'un simple «récit» présentant l'égalité comme étant la valeur primaire. Or, on pourrait tout à fait proposer un récit différent permettant d'accorder la primauté non pas à la valeur d'égalité mais plutôt à la valeur de vérité. Christiano lui-même offre d'ailleurs les éléments permettant de construire un tel récit, notamment lorsqu'il affirme: "The interest in truth is fundamental because it is the interest that gives purpose to having beliefs for rational beings" (ibid., I 54). On pourrait ainsi dire que c'est la vérité, et non l'égalité, qui est au fondement de toute autre valeur, en soulignant par exemple que, pour des êtres rationnels comme nous, l'objectif ultime est de faire sens de ce monde dans lequel nous avons été projetés contre notre gré. La valeur d'égalité pourrait alors être comprise comme découlant simplement de cette valeur de vérité plus fondamentale: les êtres humains peuvent être dits égaux dans la mesure où ils ont en commun cette quête ultime de la vérité.

On peut également concevoir des récits de différente nature permettant de considérer d'autres types de valeurs comme étant primordiales. La question urgente devient alors la suivante: comment peut-on déterminer quel récit est supérieur à l'autre? Confronté à deux récits distincts ayant chacun une cohérence interne et un attrait intuitif, on voit mal comment on pourrait parvenir à trancher entre l'un ou l'autre. En tant que reconstructions imaginaires des fondements axiologiques de l'univers politique, l'un comme l'autre paraît plausible, et opter pour l'un plutôt que pour l'autre semble être un choix purement ad hoc. Or, dans la mesure où le premier récit — fondant l'égalité — permet de fonder la supériorité de la démocratie, et où le second — fondant la vérité — permet de fonder la supériorité de l'épistocratie, et dans la mesure où l'on ne semble disposer d'aucun critère 
pour préférer l'un plutôt que l'autre, on se voit obligé de conclure qu'une telle stratégie argumentative ne permet pas de chasser le spectre épistocratique.

Peut-être existe-t-il une autre façon de fonder in abstracto la supériorité d'une valeur sur une autre en offrant un type de démonstration qui s'apparente moins à un simple récit. Si une telle démarche est possible, elle m'échappe. Quoi qu'il en soit, je préfère à présent me tourner vers une troisième stratégie afin de fonder la supériorité de la démocratie, une stratégie qui me paraît de loin être la plus prometteuse.

\section{La valeur épistémique de la démocratie}

Les stratégies précédentes se sont soldées par un échec. En tentant de discréditer la notion de vérité politique (nihilisme), on parvient certes à invalider la justification de l'épistocratie, mais on invalide par le fait même la justification de toute position ou de tout système politique, y compris la démocratie elle-même. En tentant d'interpréter la vérité politique comme un produit procédural (quasi-nihilisme), on est confronté à l'inévitabilité de faire appel à des vérités non procédurales, et on se retrouve à nouveau face au spectre épistocratique. Enfin, en tentant de démontrer in abstracto la supériorité axiologique de la valeur d'égalité afin de fonder la supériorité de la démocratie, on semble parvenir à n'offrir rien de mieux, en guise de démonstration, qu'un simple récit.

La troisième stratégie que je souhaite considérer ici consiste à nier le problème de départ en soutenant qu'il n'existe aucun véritable trade-off entre égalité et vérité. Il n'existerait pas de tel trade-off pour la simple raison que, loin de mener à un déficit épistémique, la démocratie maximiserait en réalité les chances d'atteindre de bonnes décisions politiques. Aucun autre système, aucune forme de gouverne des sages, par exemple, ne pourrait autant que la démocratie - ou plus exactement, autant qu'une démocratie bien organisée - mener aux vérités politiques.

Cette stratégie est clairement plus répandue dans la littérature que les deux précédentes et peut prendre différentes formes ${ }^{16}$. Il s'agit de l'idée principale qui sous-tend la plupart des théories "délibérativistes» contemporaines: plutôt que de concevoir que les délibérations démocratiques constituent la vérité (quasi-nihilisme de la section 2), la plupart des délibérativistes conçoivent la délibération comme étant la meilleure procédure pour parvenir aux vérités politiques, conçues comme indépendantes de la

16. Une stratégie de ce type est défendue par Thomas Christiano. En plus de tenter de démontrer la primauté axiologique de l'égalité (section 3), Christiano souligne le potentiel épistémique indéniable de la démocratie: "We have reason to think that a society that promotes public deliberation will make better decisions. [...] In short, the process of public deliberation will serve as a kind of filtering device taking out the egregious forms of ignorance regarding interests and justice» (Christiano, 2008, 194). 
délibération elle-même. L'argument déployé contre l'épistocratie consiste donc à nier que la gouverne d'une poignée de prétendus experts soit épistémiquement préférable à une procédure fondée sur la délibération démocratique. Certes, en limitant la participation citoyenne à la simple élection de représentants, les démocraties actuelles ont une valeur épistémique plutôt limitée, mais l'épistocrate s'attaque vraisemblablement à un homme de paille s'il juge de la valeur épistémique de la démocratie en se référant strictement aux démocraties représentatives contemporaines. Il suffit en effet de concevoir un design institutionnel plus raffiné, plaçant au cœur du processus politique la délibération et l'échange d'idées entre citoyens, pour saisir l'immense potentiel épistémique propre à la démocratie.

Mon but ici n'est pas de présenter les différents modèles de procédures délibératives qui ont été proposés ou testés à travers les années, et d'en évaluer la valeur épistémique. D'autres que moi se sont livrés de manière fort éclairante à une analyse de ces différents modèles et des nombreux enjeux qu'ils soulèvent ${ }^{17}$. Mon but est plutôt de déterminer en vertu de quelles caractéristiques une bonne démocratie délibérative peut être dite supérieure à tout autre système politique en tant que véhicule menant aux vérités politiques. Je me limiterai à distinguer deux grandes façons par lesquelles on peut concevoir la démocratie comme possédant un potentiel épistémique supérieur à tout autre système politique: I) en soulignant l'apport du nombre de participants à la délibération $(4 . \mathrm{I}) ; 2$ ) en soulignant la valeur ajoutée aux lois et aux décisions politiques par la procédure délibérative (4.2).

\subsection{La valeur instrumentale de la procédure démocratique}

La première stratégie est la manière la plus standard de concevoir la valeur épistémique de la démocratie. L'avantage comparatif de la démocratie, selon cette approche, tient tout simplement au nombre de ses participants potentiels, qui s'étend bien sûr à l'ensemble des citoyens. S'il est vrai que deux têtes valent mieux qu'une, des millions de têtes peuvent certainement valoir leur pesant d'or, ne serait-ce que par le nombre de points de vue, d'arguments et de contre-arguments qui peuvent être soulevés et évalués. Tout le défi consiste donc à parvenir à tirer profit de ce potentiel épistémique en développant des mécanismes qui facilitent l'échange, l'évaluation et la rétention d'idées, et qui limitent les risques d'irrationalité souvent associés aux phénomènes de masse.

On peut ainsi facilement voir en quoi une démocratie délibérative efficace rendrait inattrayant le modèle épistocratique d'une gouverne des sages; dans la mesure où la démocratie délibérative donne voix à tous les citoyens, les sages — ou l'élite intellectuelle — demeurent des joueurs à part entière

17. Voir surtout Goodin (2008). 
de ce système, et leur contribution est donc théoriquement tout aussi présente dans ce système. Or, puisque la démocratie délibérative préserve l'apport des citoyens au point de vue éclairé, l'épistocratie paraît perdre son avantage comparatif. La seule différence qui demeure entre les deux systèmes est donc celle du nombre de points de vue entendus dans le processus décisionnel, et, en se limitant au principe du «deux têtes valent mieux qu'une ", l'avantage épistémique semble clairement aller du côté du système qui ouvre le débat à l'ensemble des citoyens.

Il s'agit là de l'argument de base favorisant la thèse de la supériorité épistémique de la démocratie délibérative. Plusieurs prémisses peuvent bien sûr être mises en doute ou critiquées. On pourrait par exemple souligner que les points de vue éclairés de la société risquent fort de se perdre dans la médiocrité majoritaire, que la rhétorique supplantera inévitablement la logique et que les positions qui seront adoptées à la suite d'une délibération ouverte à tous ne seront pas les meilleures ou les plus rationnelles, mais plutôt celles défendues par les meilleurs orateurs. Si ces objections sont légitimes, on notera que, pour la plupart d'entre elles, il suffira au défenseur de la démocratie délibérative de rétorquer que la solution réside, non pas dans une restriction de la participation populaire, mais plutôt dans un meilleur encadrement de cette participation, c'est-à-dire dans un perfectionnement procédural qui favorise une présentation plus fidèle de la valeur des idées émises. Le potentiel épistémique de la démocratie délibérative demeure donc supérieur à celui de l'épistocratie, et l'accomplissement de ce potentiel devient un problème strictement empirique quant à la qualité du design institutionnel.

On pourrait bien sûr rétorquer que le problème demeure entier si aucun design empiriquement réalisable ne parvient à enrayer les différentes formes d'irrationalité collective et à soutirer le potentiel épistémique promis par la théorie. Soutenir qu'il est possible de réaliser la pleine valeur épistémique de la démocratie délibérative n'est peut-être ultimement qu'un acte de foi. Évidemment, on pourra répondre au sceptique que la croyance en l'impossibilité de réaliser le potentiel épistémique de la démocratie délibérative est tout autant un acte de foi. On aura alors l'impression d'avoir atteint un cul-de-sac.

Plutôt que de chercher une sortie à cette impasse, il est possible d'envisager une deuxième manière de concevoir la supériorité épistémique de la démocratie délibérative ${ }^{18}$.

18. Cela ne revient pas à dire que la première manière de concevoir cette supériorité épistémique échoue, mais simplement que son acceptation n'est pas nécessaire à la thèse d'une supériorité épistémique. 


\subsection{La valeur ajoutée par la procédure démocratique}

En plus de soutenir que l'inclusion de l'ensemble des points de vue de la société maximise les chances d'atteindre les bonnes décisions politiques, on peut souligner que l'inclusion de l'ensemble des points de vue augmente la valeur des décisions elles-mêmes. Autrement dit, si la première stratégie consiste à souligner la valeur instrumentale de la procédure démocratique dans l'atteinte des bonnes décisions politiques, la deuxième stratégie consiste plutôt à souligner que la procédure démocratique est elle-même un élément constitutif des bonnes décisions politiques ${ }^{19}$.

L'idée essentielle qui sous-tend une telle proposition est la suivante: le fait que les citoyens soient auteurs de leurs propres lois apporte une certaine plus-value à celles-ci. À l'inverse, une loi ou une décision politique qui est prise indépendamment des individus concernés et qui leur est ensuite imposée voit sa valeur affectée par son caractère autoritaire ou externe. Pour simplifier, on distinguera la valeur objective et la valeur subjective d'une loi ou d'une décision politique: même une décision que l'on sait être objectivement supérieure à toute autre - par exemple parce qu'elle est la meilleure façon connue de répondre à un problème donné - perd au moins une partie de son attrait lorsqu'elle nous est imposée par une tierce instance ${ }^{20}$. Nous préférerons prendre nous-mêmes les décisions qui nous concernent, au risque de passer à côté des meilleures décisions objectives, plutôt que de laisser d'autres prendre ces décisions à notre place ${ }^{21}$.

Dans cette perspective, dire que la démocratie a une valeur épistémique supérieure à tout autre système revient à dire que seule la démocratie voit ses lois ou décisions bonifiées par ce qu'on peut appeler la légitimité intrinsèque de la procédure démocratique. Cela ne veut pas dire que toutes les lois résultant d'une démocratie sont préférables à toutes les lois résultant d'une épistocratie. Des démocraties peuvent mener - et ont historiquement mené - à des atrocités, et dans de tels cas on préférera incontestablement des lois plus éclairées émanant d'un système non démocratique. Cependant, le fait qu'une loi provienne d'une bonne procédure démocratique est un élément qui y ajoute toujours une valeur positive. On peut s'inspirer de la théorie de W. D. Ross (Ross, I930) et souligner qu'être le fruit d'une bonne procédure démocratique est toujours, pour une loi ou une décision politique,

19. Cette stratégie ne doit pas être confondue avec la stratégie "quasi-nihiliste» qui conçoit les vérités politiques comme étant constituées par la procédure délibérative. La différence peut être présentée de la manière suivante: selon la stratégie quasi-nihiliste, pour que $\mathrm{X}$ soit la bonne décision politique $\mathrm{X}$ doit être choisi dans le cadre d'une bonne procédure délibérative. À l'inverse, selon la stratégie que j'expose dans cette section, $\mathrm{X}$ peut résulter d'une bonne procédure délibérative sans pour autant être la bonne décision politique, mais le fait qu'elle soit choisie dans une bonne procédure délibérative est un élément qui ajoute toujours de la valeur à une décision.

20. J'entends par tierce instance un corps décisionnel non élu ou choisi par la population.

21. Je ne prétends pas qu'il s'agisse là d'un principe absolu n'admettant aucune exception. 
un bien prima facie (ou pro tanto). Ce bien prima facie ne constitue cependant qu'un aspect de la valeur finale de la loi ou de la décision, et cet aspect peut ultimement être supplanté par d'autres aspects. Ainsi, une loi choisie démocratiquement mais aux conséquences atroces est prima facie bonne par sa nature démocratique, prima facie mauvaise pour ses conséquences atroces, et - fort probablement - globalement mauvaise en vertu du poids supérieur de sa dimension négative. Néanmoins, qu'une loi ou une décision politique soit le produit d'une bonne procédure démocratique constitue toujours un bien prima facie, une plus-value.

Je vois mal comment on pourrait nier l'existence d'une telle plus-value propre aux lois et aux décisions démocratiques. On peut tout au plus tenter d'en minimiser l'importance, en affirmant que cette valeur ajoutée est somme toute marginale, et qu'elle ne peut à elle seule fonder la supériorité épistémique de la démocratie par rapport à l'épistocratie. C'est là un argument qui me semble cependant peu convaincant. Il suffit à mon sens de considérer l'analogie avec les décisions individuelles pour voir à quel point on accorde une valeur importante à ce que j'appelle la légitimité intrinsèque. Le fait que nous soyons responsables des décisions qui nous concernent est crucial pour les êtres que nous sommes. Il en va de notre autonomie et de notre dignité. Je vois mal pourquoi ce même principe perdrait de son importance lorsqu'il est appliqué aux collectivités.

Rappelons enfin que, même si l'on est sceptique quant à l'importance de la valeur ajoutée aux lois ou aux décisions par la procédure démocratique, on peut continuer à défendre la supériorité épistémique de la démocratie en utilisant la stratégie que j'ai présentée à la section 4.I. Une critique efficace doit s'attaquer à la fois à l'argument de la valeur instrumentale et à celui de la valeur ajoutée. S'il reste des doutes quant au succès de ces deux stratégies argumentatives, j'espère tout de même avoir convaincu le lecteur que ce type de stratégie est plus prometteur que les stratégies considérées dans les sections précédentes.

\section{Conclusion: la contestation comme outil épistémique}

L'enquête à laquelle je me suis livré dans cet article avait pour point de départ la notion de vérité politique. L'existence de telles vérités politiques est reconnue implicitement par tout contestataire, ne serait-ce que parce que l'acte de contestation présuppose la croyance selon laquelle la position politique contestée n'est pas la bonne position politique. Or, s'il existe effectivement des vérités politiques, pourquoi ne devrait-on pas maximiser nos chances de les atteindre en limitant le pouvoir populaire et en évitant ainsi le déficit épistémique généralement associé à la démocratie? Tel est le spectre épistocratique auquel j'ai tenté de répondre.

Les deux premières stratégies argumentatives que j'ai envisagées ne sont pas parvenues à fonder la préférabilité de la démocratie par rapport à l'épistocratie. Alors que la tentative de discréditer ou de modifier la notion de vérité 
politique a résulté en une incapacité de justifier le système démocratique, la tentative de démontrer la supériorité axiologique de la valeur d'égalité n'a pu mener à une forme de démonstration pertinente. La troisième stratégie s'est cependant révélée plus efficace; s'il est vrai que les démocraties représentatives contemporaines comportent un déficit épistémique, le système politique qui démontre le potentiel épistémique le plus élevé demeure la démocratie, à savoir une démocratie délibérative bien organisée. La supériorité épistémique d'un tel système peut être conçue de deux manières différentes: I) elle a une valeur instrumentale, puisque le nombre élevé de participants augmente la possibilité d'atteindre les bonnes décisions politiques; 2) elle a une valeur ajoutée, puisque la procédure délibérative jouit d'une légitimité intrinsèque apportant une plus-value aux lois et aux décisions qui en découlent.

Si cette troisième stratégie est effectivement la meilleure manière de défendre la démocratie contre l'épistocratie, on peut tirer quelques leçons quant au rôle de la contestation dans la vie politique d'une société. Notons d'abord que la contestation serait un phénomène marginal, sinon inexistant dans une démocratie délibérative idéale. Dans une perspective utopiste, la société aurait des mécanismes de délibération et d'inclusion citoyenne parfaitement organisés, offrant toujours aux citoyens le moyen de faire entendre leur voix à l'intérieur des institutions. Ces institutions permettraient aux citoyens de présenter leurs désaccords dans le cadre de débats visant l'exhaustivité complète des enjeux - c'est-à-dire menant à l'atteinte de vérités politiques $^{22}$. La contestation peut donc être conçue comme le reflet d'une imperfection institutionnelle.

Quittant le terrain de la théorie idéale, on notera que, dans le cadre de nos démocraties représentatives, la contestation apporte une contribution épistémique en lien avec les deux types de valeurs que j'ai associées à la démocratie délibérative: la valeur instrumentale et la valeur ajoutée. D’une part, l'acte de contestation contribue à la possibilité d'atteindre des vérités politiques (valeur instrumentale) en alimentant le débat public et en présentant la position politique contestée comme n'étant pas la bonne. Par ses actions, le contestataire pousse le gouvernant à justifier ses positions et induit par le fait même une forme de délibération qui, bien qu'imparfaite et menée à forces inégales, augmente notre connaissance des enjeux politiques.

D'autre part, comme on l'a mentionné dans l'introduction, l'acte de contestation interpelle toujours implicitement le gouvernant sur le lien étroit qui unit volonté populaire et gouverne légitime. Ainsi, en l'absence d'une

22. Faute d'espace, je laisse de côté la critique fort pertinente selon laquelle l'exhaustivité complète des débats est inconcevable en raison de contraintes pratiques — principalement les contraintes de temps - inhérentes à la vie politique. Tôt ou tard, on doit clore le débat, sans qu'on n'ait pu faire entièrement le tour de la question. Pour cette raison, même dans un système idéal, des citoyens pourraient juger ne pas avoir eu la possibilité d'exposer entièrement leurs positions et dès lors avoir recours à la contestation. 
bonne démocratie délibérative, la contestation agit comme un rappel que les décisions politiques qui ne reflètent pas les positions de la population perdent par conséquent une partie de leur valeur; en effet, elles ne bénéficient pas de la valeur ajoutée découlant du fait que ce sont les individus concernés qui prennent eux-mêmes les décisions les concernant — ce que j'ai appelé la légitimité intrinsèque ${ }^{23}$.

La contestation politique constitue donc un double outil épistémique. Si son apport au débat public est indéniable, elle n'élimine cependant guère le besoin de perfectionner nos institutions afin que les points de vue des citoyens soient mieux intégrés et puissent contribuer plus directement au bien commun. S'il est vrai que la démocratie a un potentiel épistémique supérieur à tout autre système politique, nous serions bien bêtes de ne pas en tirer parti ${ }^{24}$.

\section{Bibliographie}

Ackerman B. et J. Fishkin. Deliberation Day, Yale, Yale University Press, 2004.

Audi, R. "Intuition, Inference, and Rational Disagreement in Ethics ", Ethical Theory and Moral Practice, vol. II (5), 2008, 475-492.

Ayer, A. J., 1936. Language, Truth, and Logic. Londres, Penguin Classics, chapitre VI: "Critique of Ethics and Theology", p. I I 5-I 30 .

Barry, B. Theories of Justice, Berkeley, University of California Press, I989.

Christiano, T. The Constitution of Equality: Democratic Authority and its Limits, New York, Oxford University Press, 2008.

Darwall, S., A. Gibbard et P. Railton. "Towards Fin de siècle Ethics: Some Trends", The Philosophical Review, vol. IоI, I992, p. II 5-189.

Estlund, D. Democratic Authority: A Philosophical Framework, Princeton, Princeton University Press, 2008.

"The Insularity of the Reasonable: Why Political Liberalism Must Admit the Truth", Ethics, vol. I08, nº 2, I998, p. 252-275.

Goodin, R. Innovating Democracy: Democratic Theory and Practice after the Deliberative Turn, New York, Oxford University Press, 2008.

Habermas, J. Truth and Justification, Cambridge, MIT Press, chapitre VI: «Rightness Versus Truth: on the Sense of Normative Validity in Moral Judgments and Norms", 2005, p. 237-276.

Mackie, J. Ethics: Inventing Right and Wrong, Viking Press, 1977.

McDowell, J. Mind, Value, and Reality, Cambridge, Harvard University Press, I998. Rawls, J. «Justice as Fairness: Political, not Metaphysical», Philosophy and Public Affairs, vol. I4, $\mathrm{n}^{\circ}$ 3, 1985, p. 223-25 I.

Scanlon, T. M. What We Owe to Each Other, Cambridge, Belknap Press, I999.

23. On peut, à juste titre, souligner que la démocratie représentative préserve une partie de cette valeur ajoutée. En effet, dans la mesure où ce sont les individus concernés (c'est-à-dire les citoyens) qui choisissent les représentants prenant les décisions pour eux, la démocratie représentative préserve une partie de cette valeur ajoutée, puisque ce sont indirectement les individus concernés qui prennent les décisions les concernant.

24. Un exemple original de réformes promettant de tirer parti du potentiel épistémique de la démocratie est proposé par Bruce Ackerman et James Fishkin dans Deliberation Day (Ackerman et Fishkin, 2004). 